\title{
Game Theory DVB-RCS2 Return Link Fairness Optimization Based on Timeslot Information Payload
}

\author{
Pedro H. A. Trindade, Leonardo Aguayo, Alexander Wyglinski and Renato F. Iida
}

\begin{abstract}
This paper introduces a novel algorithm modification for the conventional game theory-based time slot assignment method applied to the Digital Video Broadcast Return Channel via Satellite (DVB-RCS) system. The procedure considers the spectral efficiency as a weighting parameter for the resulting convex optimization problem of the Nash Bargaining Solution. This approach guarantees the fulfillment of Quality of Service (QoS) constraints while maintaining a higher fairness measure; results show a $5 \%$ improvement in fairness, with a $73 \%$ decrease in the standard deviation between frames, also managing to reach a $12.5 \%$ increase in individual terminal capacity distribution satisfaction.
\end{abstract}

Keywords-DVB-RCS2, Satellite Communications, Game Theory, Nash Bargaining Solution, MF-TDMA Optimization.

\section{INTRODUCTION}

Satellite networks are expected to play a vital role in 5G and beyond communications systems, especially in the context of hybrid terrestrial-satellite networks. Optimization of radio resources in the uplink is fundamental to coping with data rate requirements within a machine-to-machine (M2M) scenario. The ETSI Digital Video Broadcasting-Satellite (DVB-S) standard presents advances for the forward link with DVB-S2X [1] via new modulation and coding (MODCOD) schemes with high spectral efficiency. For the return link, DVB-RCS2 [2, 3] offers an advanced and flexible configuration for multiuser access Multi-Frequency Time Division Multiple Access (MFTDMA) framework. This work addresses the optimization of multiuser allocation in the MF-TDMA structure considering different user priorities and the MODCOD options allowed by the RCS 2 standard.

In Section II, we give an elementary description of the satellite network elements, focusing on the DVB-RCS2 return link timeslot (TS) assignment; in Section III we discuss the previous studies in satellite networks frame optimization, then in Section IV we describe the mathematical model for the previously specified variables. Section V details the Nash bargaining solution for the game theory distribution of resources in a DVB-RCS2 frame, informing our proposed modifications; Section VI refers to all implementation tools used and the essential parameters for replication. Finally, sections VII and VIII presents the results and conclusions for the simulated scenario, highlighting further development.

\section{SySTEM OVERVIEW}

The DVB-RCS2 specifies a network control center (NCC) that communicates through a satellite relay with multiple return channel terminals (RCSTs). In the forward link, the
NCC periodically sends messages to the RCSTs, such as the MF-TDMA structure and the MODCOD schemes, based on the RCSTs channel conditions. The RCSTs respond with packet bursts in their allowed time and frequency slots within the MF-TDMA structure. RCS2 allows Adapting Code and Modulation (ACM) and Dynamic Rate Adaption (DRA) [4, 5] to improve spectral efficiency. RCST's transmission parameters, such as code rate, modulation order, and symbol rate, are based on NCC's estimates of signal to noise plus interference ratio (SNIR). It is a bandwidth-on-demand solution, where the NCC must calculate the number of resources to assign to each corresponding terminal, characterizing a Demand Assigned Multiple Access (DAMA). RCS2 allows four different types of assignments:

- Constant Rate Assignment (CRA): Requires a constant rate, based on the Service Level Agreement (SLA) between the RCST and the service provider.

- Rate Based Dynamic Capacity (RBDC): The RCST requests a certain bit rate constraint to be met that remains effective until updated.

- Volume-Based Dynamic Capacity (VBDC): The RCST use this capacity request when it knows the amount of data to transmit, making a request based on the volume of data and not on data rate.

- Free Capacity Assignment (FCA): In this modality, without an RCST request, the NCC assigns the surplus of capacity not allocated in the current scheduling iteration.

Here, we consider allocating the superframe (SF) structure inside the MF-TDMA hierarchy, where the BTU (bandwidthtime unit) is the minimum allocation unit in the time-frequency grid. An RCST is assigned to a timeslot (TS) composed of one or more BTUs. Different MODCODs require TSs of different sizes due to their different payloads.

RBDC and the VBDC are the only types of requests in which there is a possibility of defining a scheduling strategy that optimally assigns users in an SF. Optimal in this context means that (i) the allocation is fair in the sense of the Jain fairness index as described in Eq. (4); (ii) the QoS requirements are met, and (iii) the BTU resources are not being wasted. We look for optimal allocation algorithms, having both a fair distribution to each RCST and a feasible datarate efficiency while maintaining low operational complexity. Computational speed is relevant because the NCC must broadcast the complete specification of RSCT's TSs through the Terminal Burst Time Plan Table (TBTP) at every SF. Typical 
SF duration is between $100 \mathrm{~ms}$ and $1 \mathrm{~s}$, e.g. $256 \mathrm{~ms}$ in [6].

\section{RELATED WORK}

Different approaches were considered to optimize the MFTDMA resource allocation in DVB-S systems, such as optimizing power allocation in multi-beam satellites [7]. References $[8,6]$ present a game-theoretic approach to solving the Nash bargaining problem with Lagrange multipliers. [9] employs a buddy-fit algorithm and [10] proposes an algorithm to analyze the spatial constraints and interference patterns in the satellite configuration.

This paper relies on a different approach on the gametheoretic solution, solving the general problem described in [8] using RCST's SNIR, which defines its MODCOD and thus its information payload per BTU (2). The strategy leads to a means to prioritize the resource distribution in the dimensions of the SF, concerning not only the minimum requirements of quality of service (QoS) but also optimizing fairness.

\section{SySTEM MODELING}

This section describes the modeling aspects of the DVBRCS2 implementation, focusing on the notation of necessary parameters and simplifying assumptions.

To simulate the birth and death of RCSTs, the ones attempting to enter the network are organized in a queue. Arrivals are modeled as a Poisson random variable, with parameter $\lambda$ as the mean arrival rate per SF in the system, which defines the system traffic intensity, affecting the resource distribution performance.

Assigned to the $i$-th RCST ( $i \in\{0,1, \cdots, N-1\}$ ), there is a tuple of parameters: a volume of data $V_{i}$ to deliver before leaving the system; a minimum required data rate to meet the QoS requirements $d_{i}$ (in BTUs/s per SF); an expected data rate $D_{i}$ (also in BTUs/s per SF); a burst length $w_{i}$ (BTUs); an allocation priority $p_{i}$; and an information efficiency $\eta_{i}$ (bits/symbol), defined by a finite set of waveform IDs in [11, pg.179]. We also consider the time duration of an SF to be 1 second. This way, $d_{i}$ and $D_{i}$ are numerically equal to the number of BTUs allocated in an SF, while using the relative measurement of an SF period $T_{S F}$ in BTUs/s.

An SF is modeled as a grid of $N_{S F}=T_{S F} \times K_{S F}$ BTUs. The $i$-th allocated RCST will receive $\phi_{i}$ BTUs per SF, no less than the minimum required $d_{i}$ and no more than the expected $D_{i}$. The number of allocated users is determined by the maximum number of allowable RCSTs in an SF that still meet the minimum QoS requirement $d_{i}$. Assuming that an RCST can only use one frequency channel concurrently, the maximum resource allocation is limited by $T_{S F}$ in the time domain of the SF.

Also, RCSTs are served in order of arrival: if an RCST is not allocated in the current SF, it maintains its position in the queue until eventually be served in the following SF.

The expected BTU rate $D_{i}$ is modeled as $D_{i}=\alpha d_{i}$, where $\alpha \in\left[1, \alpha_{\max }\right]$ is a truncated Gaussian random variable, with parameters $(\mu, \sigma)$ and $\alpha_{\max }=T_{S F} / d_{i}$, which was a design choice to emulate a real case scenario, as the proposed algorithm performance depends on the dispersion of the $D_{i}$ distribution. The $\alpha_{\max }$ value is such that the expected BTU rate does not surpass the maximum possible allocation in a ACM channel. This formulation models a random data demand of bandwidth by the terminals. Parameter $\alpha$ has distribution

$$
p(\alpha)=\left\{\begin{array}{l}
\frac{1}{\hat{p} \sqrt{2 \pi} \sigma} \exp \left[-\frac{(\alpha-\mu)^{2}}{2 \sigma^{2}}\right], \quad 1 \leq \alpha \leq \alpha_{\max }, \\
0, \quad \text { otherwise }
\end{array}\right.
$$

where $\hat{p}$ is a normalization factor such that $\int_{1}^{\alpha_{\max }} p(\alpha) d \alpha=1$. With this truncation, in general $E[\alpha]=\bar{\alpha} \neq \mu$, but we have considered a range of values $\mu$ and $\sigma$ such that the difference can be disregarded.

We also consider the total useful information per BTU

$$
q_{i}=\eta_{i} \times T_{\mathrm{BTU}}
$$

in bits per BTU, where $T_{\mathrm{BTU}}$ is the length of a BTU in symbols. The value $q_{i}$ is the amount of allocated BTUs that carries data information bits, disregarding control/redundancy bits. The information payload of the allocation is thus $\phi_{i}^{*}=$ $q_{i} \phi_{i}$, as depicted in Fig.1.

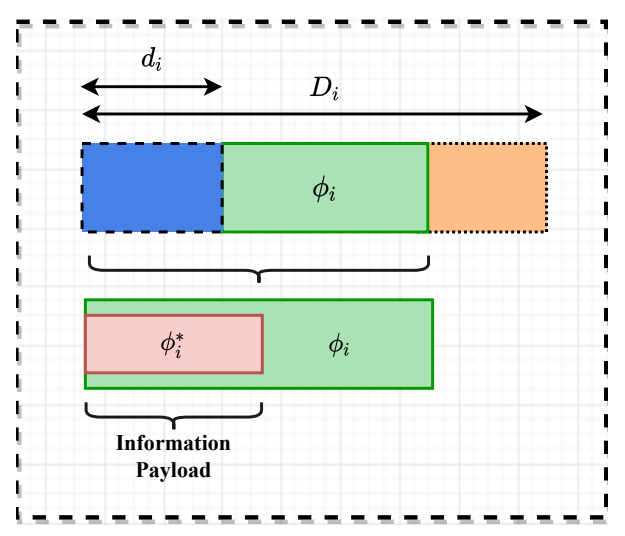

Fig. 1. Allocation range $\left(d_{i}, D_{i}\right)$ and the information payload $\phi_{i}^{*}$ in a TS.

When we take into account all the RCSTs, we define the allocated resource distribution vector in an $\mathrm{SF}$ as

$$
\phi \triangleq\left(\phi_{0}, \phi_{1}, \cdots, \phi_{N-1}\right), \phi_{i} \in \mathbb{R} \geq 0 .
$$

Fig. 2 illustrates the SF when filled by $\phi$. The $\phi_{i}$ allocated to an RCST is always a multiple $N_{i}$ of the BTU size $T_{\mathrm{BTU}}$. In frequency, it occupies a fixed multiple $L_{i}$ of the BTU bandwidth $B_{\mathrm{BTU}}$.

The fairness of an allocation can be measured with the Jain fairness index [12]

$$
J(\phi)=\frac{\left(\sum_{i=0}^{N-1} \frac{\phi_{i}}{D_{i}}\right)^{2}}{N \sum_{i=0}^{N-1}\left(\frac{\phi_{i}}{D_{i}}\right)^{2}}=\frac{\left(\sum_{i=0}^{N-1} \hat{\phi}_{i}\right)^{2}}{N \sum_{i=0}^{N-1} \hat{\phi}_{i}^{2}},
$$

where $\hat{\phi}_{i}=\frac{\phi_{i}}{D_{i}}$, the normalized BTU distribution, is the ratio between the capacity an RCST requests and the capacity it receives. $\hat{\phi}_{i}$ can be interpreted as a measurement of the satisfaction an RCST experiences after receiving $\phi_{i}$ BTUs from the NCC's scheduler. The Jain fairness index measures then the perceived average fairness of the resource allocation, 


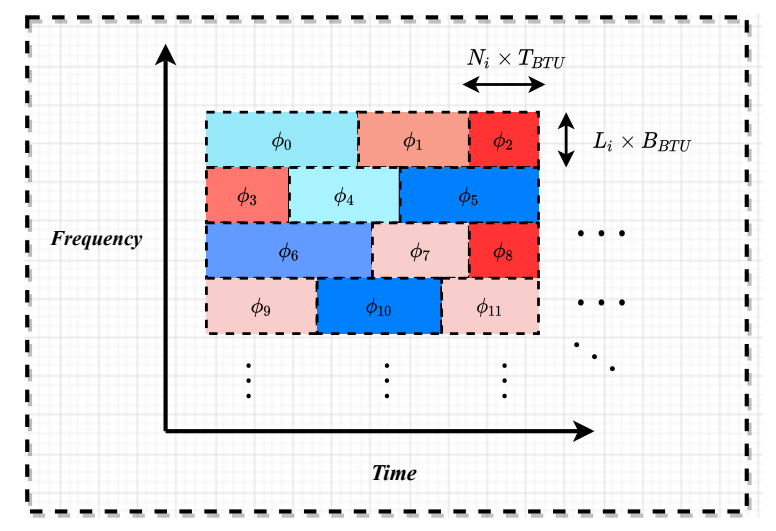

Fig. 2. Distribution of BTU resources $\phi_{i}$ in a DVB-RCS2 SF. All $\phi_{i}$ occupies the same bandwidth in an SF.

and it is dependent on the coefficient of variation of a given distribution vector. There is a large body of literature about fairness metrics [13] and different ways to construct a custom fairness measure [14] to highlight certain aspects of a given distribution. We chose the Jain index as the benchmarking fairness metric as a means to suit with past analysis of the game theory bargaining solution for resource allocation in a satellite communication system.

\section{Proposed Framework}

When solving resource allocation problems involving multiple receivers in a cooperative setting, the Nash bargaining solution is often employed $[15,16,8,6]$, which is an objective function in game theory that satisfies both Pareto efficiency and proportional fairness [17, 18, 13], meaning a distribution solution that allows for both an efficient distribution as also a reasonable, fair one.

This section describes the mathematical framework for the Nash bargaining solution applied to a TS allocation in an MFTDMA SF, which is comprised of three main aspects: (i) The predefined Nash solution function that solves the game theory bargaining problem; (ii) the constraints of the SF dimensional limitations and (iii) the application of the method of Lagrange multipliers with Karush-Kuhn-Tucker (KKT) conditions for solving the consequent convex optimization problem. Lastly, in this section, we describe our modifications of this framework, aiming to optimize the fairness measure at each SF.

Interpreting the RCST resource scheduling dilemma as a game theory bargaining problem, each $\phi_{i}$ in Eq. (3) is understood as an utility function. To solve this bargaining problem the Nash bargaining solution is employed. The objective function for the weighted Nash bargaining solution is

$$
\mathcal{N}_{B}(\boldsymbol{\phi}, \boldsymbol{p})=\prod_{i=0}^{N-1} \phi_{i}^{p_{i}},
$$

where $p_{i}$ is a priority weight ascribed to each RCST. A larger $p_{i}$ means a higher priority in the allocation i.e., more BTUs proportionally to the other RCSTs at the optimal assignment.

It is possible to modify the objective function (5) into a convex function by employing a logarithmic transformation, and thus the problem become a convex optimization problem. The full statement using the defined variables therefore becomes

$$
\begin{aligned}
& \max _{\phi} \sum_{i=0}^{N-1} p_{i} \ln \phi_{i} \\
& \text { s.t. } \phi_{i} \leq D_{i} \quad \forall i, \\
& \phi_{i} \geq d_{i} \quad \forall i, \\
& \phi_{i} \geq 0 \quad \forall i, \\
& \sum_{i=0}^{N-1} \phi_{i} \leq N_{S F} .
\end{aligned}
$$

Employing the method of Lagrange multipliers, the Lagrangian function for Eq. (6) can be written as

$$
\begin{aligned}
\mathcal{L}(\phi) & =\sum_{i} p_{i} \ln \phi_{i}+\kappa\left(N_{S F}-\sum_{i=0}^{N-1} \phi_{i}\right) \\
& +\sum_{i} k_{i}\left(\phi_{i}-d_{i}\right)+\sum_{i} K_{i}\left(D_{i}-\phi_{i}\right),
\end{aligned}
$$

where $\kappa, k_{i}$ and $K_{i}$ are non-negative Lagrange multipliers. The choice for $\phi_{i}$ is optimal when $\frac{\partial \mathcal{L}}{\partial \phi_{i}}=0$, that is

$$
\frac{\partial \mathcal{L}(\phi)}{\partial \phi_{i}}=0=\frac{p_{i}}{\phi_{i}}-\kappa+k_{i}-K_{i}
$$

with the Karush-Kuhn-Tucker (KKT) conditions, that results from the inequality constraints in Eq. (6), being

$$
\begin{aligned}
k_{i}\left(d_{i}-\phi_{i}\right) & =0, \\
K_{i}\left(D_{i}-\phi_{i}\right) & =0 .
\end{aligned}
$$

From conditions (14) applied in (12) and solving for $\phi_{i}$ following the steps at $[8,6]$, we get

$$
\phi_{i}= \begin{cases}p_{i} v, & \text { if } d_{i}<p_{i} v<D_{i}, \\ d_{i}, & \text { if } p_{i} v \leq d_{i}, \\ D_{i}, & \text { if } D_{i} \leq p_{i} v,\end{cases}
$$

where $v=1 / \kappa$ is a chosen constant such that the constraint $\sum_{i=0}^{N-1} \phi_{i} \leq N_{S F}$ is met. This solution resembles the solution for the water filling problems $[19,20]$ that often appears in wireless communication papers.

We propose to define the priority weights in the general Nash bargaining solution convex problem formulation (6) as $p_{i}=1 / q_{i}$, where $q_{i}$ is the total useful information payload per BTU, defined previously in (2). This modification changes the solution so that the algorithm considers the useful capacity gained by an RCST, with every BTU, knowing that an RCST with a higher SNIR has a larger information payload per burst and thus gains more utility per BTU.

This modification guarantees an overall fairer approach, as the legacy solution with all equal priorities allocates the surplus equitably in terms of BTUs but not in terms of endpoint capacity that an RCST receives.

Notice that although (6) is a real convex optimization problem, meaning it can output solutions in all $\mathbb{R}^{+}$, the solution in the DVB-RCS2 SF demands an integer allocation, $\phi_{i}$ must also be a multiple of the burst length $\omega_{i}$. 
An integer solution can be obtained with a rounding process described in [6], where the fractional part of each $\phi_{i}$ is added i.e.

$$
\phi_{\text {freed }}=\left\lfloor\sum_{i=0}^{N-1}\left(\phi_{i}-\left\lfloor\phi_{i}\right\rfloor\right)\right\rfloor,
$$

where $\lfloor$.$\rfloor is the floor function and \phi_{\text {freed }}$ is distributed to each RCSTs in order of priority $p_{i}$, a burst length $w_{i}$ at a time to each RCST, until all BTUs are allocated. As the amount of resources allocated are way larger than $\phi_{\text {freed }}$, the loss in optimality by this rounding process is minimal and thus can be disregarded.

\section{Simulator AND SCENARIO DESCRIPTION}

For employing and validating the proposed algorithm in a realistic scenario, we developed an event-driven DVBRCS2 simulator using Python language. When the eventdriven model is discrete [21], time advances as a new system transition occurs. Our model is thus defined as a discreteevent system (DES), and the simulator uses the Python library Simpy [22] to control this DES queue behavior. The scheduler registers all the RCSTs allowed to transmit data and exactly when each RCST will send this data. At the beginning of the transmission, a new event is then triggered, and the error probability is calculated to evaluate the packet error rate. The RCST link-layer behavior was implemented, meeting the DVB-RCS2 standard definitions rigorously.

The simulator utilizes geospatial data to calculate a detailed link budget for each RCST, using a satellite $G / T$ footprint map as an input parameter. For each traffic load point, a number of $N_{d}$ simulations, or drops, is performed to obtain statistics of all measurements. For each simulation drop, the RCSTs are randomly distributed in a map, where, for the intents of this paper, we chose the country of Brazil as the benchmarking location. Each point in the map has a birth probability of receiving a new RCST, proportional to the population density of this specific area. This is used to simulate the contrast between high-density populated areas and less populated areas, with different satellite $G / T$ values.

To solve the convex optimization problem defined by the Nash bargaining solution, we used the library CVXPY [23]. For comparison purposes, we implemented a reference scheduler to allocate the BTUs using the traditional game theory allocation algorithm that has all the priorities equal for each RCST. Base results are compared with the new algorithm described previously, in which the BTU allocation is weighted by the $p_{i}$ parameter, inversely proportional to the information payload per BTU $q_{i}$.

All the necessary parameters to replicate the experiment are available in table I. The simulation ends after $T_{\text {sim }}=100 \mathrm{SFs}$, where the average total number of RCSTs served in a drop is $N_{\text {served }}=\lambda T_{\text {sim }}$, serving $\approx 5.000$ RCTs at the last $\lambda$ simulation point.

\section{RESULTS}

In Fig. 3 we present a comparison of the performance of the two algorithms using as a metric the Jain fairness index. It is possible to verify that the proposed algorithm demonstrates
TABLE I

TABLE OF SIMULATION PARAMETERS.

\begin{tabular}{|c||c|c|}
\hline Parameter & Description & Value \\
\hline \hline$T_{\text {Sim }}$ & simulation time in SFs & 100 \\
\hline$T_{S F}$ & Time of an SF in BTUs & 462 \\
\hline$N_{S F}$ & Number of BTUs in an SF & 2772 \\
\hline$\mu$ & $\alpha$ distribution mean parameter & 2 \\
\hline$\sigma$ & $\alpha$ distribution std. dev. parameter & 0.5 \\
\hline$T_{\mathrm{BTU}}$ & Number of symbols in a BTU & 270 \\
\hline$d_{\min }$ & Min. acceptable data rate for an RCST & $10 \mathrm{kbps}$ \\
\hline$N_{d}$ & Number of simulation drops & 10 \\
\hline$V_{i}$ & Volume of data for an RCST to transmit & $35 \mathrm{kbps}$ \\
\hline
\end{tabular}

an enhancement both in the average Jain index value, reaching a $5 \%$ gain at its peak (averaged among all SFs) and a $73 \%$ decrease in standard deviation, meaning a more stable and optimized solution. In all load cases, results were closer to the theoretical maximum of 1 .

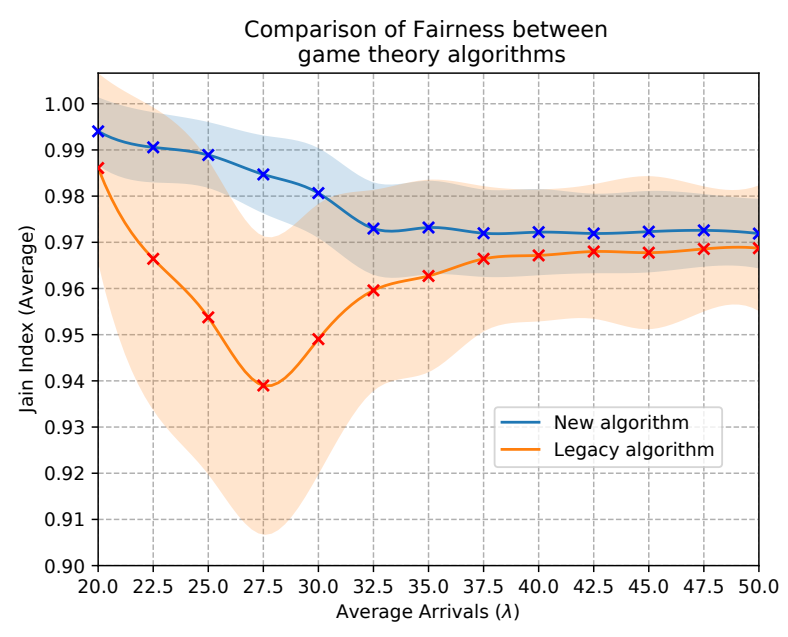

Fig. 3. Fairness performance comparison between game theory algorithms.

The simulation has a larger difference in the domain interval $22.5 \lambda \sim 35.0 \lambda$, a range in which the scheduler strategy shows effectiveness. For low values of $\lambda$, the MF-TDMA grid has enough resources to distribute among all RCSTs. The queue is under-saturated, and the scheduler is able to allocate all the SF resources. The allocation vector $\phi$ reaches maximum fairness, as each RCST receives exactly the amount of BTUs that it expects. On the other hand, when the queue is over-saturated with high values of load, the scheduler can only allocate the minimum value $d_{i}$ to each RCST in the order of arrival. In this case, there is no space for different allocation strategies to be effective, explaining the asymptotic graph behavior after the $37.5 \lambda$ mark in both graphs. Between the extreme cases, we can verify the benefits of using the proposed algorithm.

Beyond the analysis of Jain's index, we also directly evaluated the normalized BTU distribution $\hat{\phi}_{i}$ obtained for each algorithm. At Fig. 4, each point is the average $\hat{\phi}_{i}$ value among all RCSTs. The maximum value falls in the same load range of Fig.3, with an improvement of $\approx 12.5 \%$ in the $30 \lambda$ mark. 


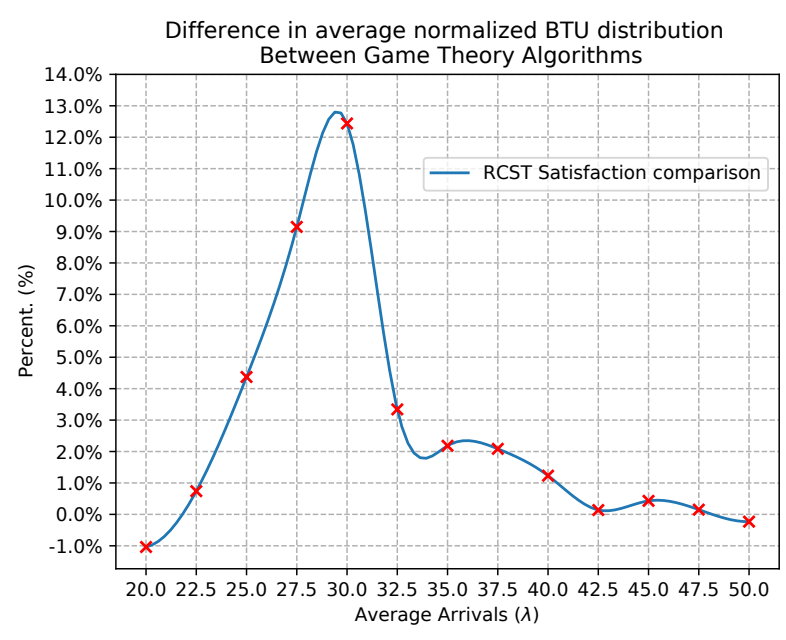

Fig. 4. Difference in average normalized BTU distribution between legacy and proposed algorithm.

\section{DISCUSSION AND CONCLUSION}

In this paper, we proposed a modification to the traditional game theory framework for capacity resource allocation in a DVB-RCS2 MF-TDMA SF, considering the information payload $q_{i}$ on each waveform ID to weigh the priority parameters in the convex optimization problem that arises from the Nash bargaining solution. The new procedure achieved better performance when compared to the traditional formulation, suggesting its employment in new scenarios.

A first extension is to apply the same priority strategy to multi-beam satellites, where a second priority would be assigned to each beam. A second possibility is the investigation of scenarios with larger SF lengths to accommodate a massive number of users (in the order of $10^{6}$ ) with low traffic density for ultra-reliable communications where the high latency of the GEO satellite link is not a problem. Finally, the insertion of LEO orbits in the simulator to take into account the Doppler effect and verify the algorithm's behavior in a dynamic SINR environment.

\section{REFERENCES}

[1] ETSI EN 302 307-2. "Digital Video Broadcasting (DVB);Second generation framing structure, channel coding and modulation systems for Broadcasting, Interactive Services, News Gathering and other broadband satellite applications; Part 2: DVB-S2 Extensions (DVB-S2X)," in: 2020.

[2] ETSI EN 301 545-2. "Digital video broadcasting (DVB); second generation DVB interactive satellite system (DVBRCS2); part 2: lower layers for satellite standard," in: 2014.

[3] ETSI EN 301 545-4. "Digital video broadcasting (DVB); second generation DVB interactive satellite system (DVBRCS2); part 4: guidelines for implementation and use of EN $301545-2$," in: 2014.

[4] M. A. V. Castro, L. S. Ronga, and M. Werner. "Dynamic Rate Adaptation (DRA) and Adaptive Coding and Modulation (ACM) efficiency comparison for a DVB-RCS system". In: 2005 2nd International Symposium on Wireless Communication Systems. 2005, pp. 822-826.

[5] M. Angelone et al. "Performance of a combined dynamic rate adaptation and adaptive coding modulation technique for a DVB-RCS2 system". In: 2012 6th Advanced Satellite Multimedia Systems Conference (ASMS) and 12th Signal Processing for Space Communications Workshop (SPSC). 2012, pp. 124-131.

[6] Gonzalo Seco-Granados et al. "Algorithm for Fair Bandwidth Allocation with QoS Constraints in DVB-S2/RCS." In: Jan. 2006.

[7] Yang Hong et al. "Optimal power allocation for multiple beam satellite systems". In: Feb. 2008, pp. 823-826. DOI: $10.1109 /$ RWS .2008.4463619.

[8] Antoni Morell, Gonzalo Seco-Granados, and Angeles Vazquez-Castro. "SAT03-3: Joint Time Slot Optimization and Fair Bandwidth Allocation for DVB-RCS Systems". In: Jan. 2007, pp. 1-5. DOI: 10.1109/GLOCOM.2006.517.

[9] T. Zhang, Q. Dong, and J. Zhang. "MF-TDMA Optimal Timeslot Allocation Algorithm". In: 2008 China-Japan Joint Microwave Conference. 2008, pp. 824-828.

[10] S. Alouf et al. "Quasi-optimal bandwidth allocation for multispot MFTDMA satellites". In: Proceedings IEEE 24th Annual Joint Conference of the IEEE Computer and Communications Societies. Vol. 1. 2005, 560-571 vol. 1.

[11] ETSI. "TR 101 545-4 - V1.1.1 - Digital Video Broadcasting (DVB); Second Generation DVB Interactive Satellite System (DVB-RCS2); Part 4: Guidelines for Implementation and Use of EN 301 545-2". In: 1 (2014)

[12] Raj Jain, Dah Ming Chiu, and Hawe WR. "A Quantitative Measure Of Fairness And Discrimination For Resource Allocation In Shared Computer Systems". In: CoRR cs.NI/9809099 (Jan. 1998).

[13] Huaizhou SHI et al. "Fairness in Wireless Networks:Issues, Measures and Challenges". In: IEEE Communications Surveys Tutorials 16.1 (2014), pp. 5-24. DOI: 10.1109 / SURV . 2013.050113 .00015$.

[14] Tian Lan et al. "An Axiomatic Theory of Fairness in Network Resource Allocation". In: 2010 Proceedings IEEE INFOCOM. 2010, pp. 1-9. DOI: 10 . 1109 / INFCOM . 2010 . 5461911.

[15] Holger Boche and Martin Schubert. "Nash Bargaining and Proportional Fairness for Wireless Systems". In: IEEE/ACM Transactions on Networking 17.5 (2009), pp. 1453-1466. DOI: 10.1109/TNET.2009.2026645.

[16] Ravi Mazumdar, L.G. Mason, and Christos Douligeris. "Fairness in Network Optimal Flow Control: Optimality of Product Forms". In: Communications, IEEE Transactions on 39 (June 1991), pp. 775-782. DOI: $10.1109 / 26.87140$.

[17] F.P. Kelly. "Charging and Rate Control for Elastic Traffic". In: European Transactions on Telecommunications 8 (Feb. 1997). DOI: 10.1002 /ett. 4460080106 .

[18] Li Bin Jiang and Soung Chang Liew. "Proportional fairness in wireless LANs and ad hoc networks". In: IEEE Wireless Communications and Networking Conference, 2005. Vol. 3. 2005, 1551-1556 Vol. 3. DOI: 10.1109 / WCNC . 2005. 1424745 .

[19] Daniel Palomar and Javier Fonollosa. "Practical algorithms for a family of waterfilling Solutions". In: Signal Processing, IEEE Transactions on 53 (Mar. 2005), pp. 686-695. DOI: 10 . 1109/TSP.2004.840816.

[20] Qilin Qi, Andrew Minturn, and Yaoqing Yang. "An efficient water-filling algorithm for power allocation in OFDM-based cognitive radio systems". In: 2012 International Conference on Systems and Informatics (ICSAI2012). 2012, pp. 20692073. DOI: 10.1109 /ICSAI. 2012.6223460.

[21] Christos G. Cassandras and Stéphane Lafortune. Introduction to discrete event systems. Springer US, 2008, pp. 1-771. ISBN: 9780387333328. DOI: $10.1007 / 978-0-387-68612-7$.

[22] SimPy Documentation. https://simpy.readthedocs. io/en/latest/.

[23] CVXPY Documentation. https://www. cvxpy.org. 